\title{
Mastofauna terrestre da Reserva Biológica Estadual do Sassafrás, Doutor Pedrinho, Santa Catarina, Sul do Brasil
}

\author{
Fernando Rodrigo Tortato ${ }^{1 *}$ \\ André Filipe Testoni ${ }^{2}$ \\ Sérgio Luiz Althoff ${ }^{3}$ \\ ${ }^{1}$ Panthera, Caixa Postal 3203, CEP 78060-970, Cuiabá - MT, Brasil \\ ${ }^{2}$ Ecoama Consultoria e Assessoria Ambiental Ltda \\ Rua Silvano Candido da Silva Sênior, 245, Sala 03, CEP 89050-280, Blumenau - SC, Brasil \\ ${ }^{3}$ Fundação Universidade Regional de Blumenau \\ Laboratório de Biologia Animal, Departamento de Ciências Naturais \\ Rua Antônio da Veiga, 140, CEP 89012-900, Blumenau - SC, Brasil \\ * Autor para correspondência \\ ftortato@hotmail.com
}

Submetido em 26/11/2013

Aceito para publicação em 21/05/2014

\section{Resumo}

De agosto de 2004 a março de 2008, foi realizado um inventário da mastofauna terrestre da Reserva Biológica Estadual do Sassafrás para aumentar o conhecimento acerca deste grupo no estado de Santa Catarina. As espécies foram registradas através de armadilhas fotográficas, avistamentos, coleta de animais mortos, rastros e armadilhas de captura viva. Foram registradas 43 espécies pertencentes a oito ordens. Informações sobre a biologia das espécies registradas foram incluídas, bem como as ameaças para os mamíferos terrestres da região.

Palavras-chave: Conservação; Espécies ameaçadas; Inventário; Mamíferos terrestres; Mata Atlântica

\section{Abstract}

A survey of the terrestrial mammals of the State Biological Reserve of Sassafrás, Santa Catarina, southern Brazil. A terrestrial mammal inventory was conducted in the State Biological Reserve of Sassafrás, Santa Catarina from August 2004 to March 2008. The objective of the study was to investigate and better describe the mammalian fauna of Santa Catarina state. We recorded species presence via several methods, including sightings, collection of fallen stock, identification of tracks, utilization of camera traps, and live animal trapping. In full, we recorded 43 mammalian species distributed among eight orders. Our constructed inventory includes information on the biology of some species recorded, as well as potential threats to the conservation of terrestrial mammals in this region.

Key words: Atlantic Forest; Conservation; Endangered species; Inventory; Terrestrial mammals 


\section{Introdução}

A perda e a fragmentação de habitat, decorrente de atividades humanas, constituem uma das maiores ameaças aos mamíferos terrestres no Brasil (COSTA et al., 2005), principalmente as espécies com distribuição no bioma Mata Atlântica (CHIARELLO, 1999; CANALE et al., 2012). No estado de Santa Catarina, que é inserido exclusivamente dentro do bioma Mata Atlântica, restam atualmente $17,46 \%$ de florestas nativas preservadas (MEDEIROS, 2002). Os resultados desta perda de habitat, associados com outras ações como a caça predatória, já ocasionaram a extinção de espécies de grande porte em grandes porções de seu território, como a Panthera onca, que tem seus últimos registros em Santa Catarina próximos à divisa com o Paraná na região da Serra do Mar (CIMARDI, 1996, MAZZOLLI, 2008).

Em relação à distribuição da mastofauna, a região sul é uma das menos conhecidas do Brasil (CÁCERES et al., 2007), sendo Santa Catarina o estado que apresenta menos publicações científicas sobre a diversidade das espécies de mamíferos com distribuição em seu território (BRITO et al., 2009), faltando principalmente informações sobre as espécies presentes em Unidades de Conservação (UC) continentais. Entre as UC de Santa Catarina, somente são encontrados trabalhos realizados na Floresta Nacional de Três Barras, no planalto norte do estado (WALLAUER et al., 2000), Reserva Particular do Patrimônio Nacional Volta Velha, no litoral norte do estado (QUADROS; CÁCERES, 2001) e no Parque Estadual Serra do Tabuleiro (CHEREM et al., 2011). Segundo Cherem et al. (2004), Santa Catarina possui 152 espécies de mamíferos com ocorrência confirmada e 60 espécies de possível ocorrência. Este elevado número de espécies a serem confirmadas para o estado evidencia a necessidade de levantamentos de longo prazo para haver um melhor conhecimento sobre a riqueza de espécies.

O objetivo deste trabalho é apresentar uma listagem das espécies de mamíferos terrestres da Reserva Biológica Estadual do Sassafrás, na região do vale do Itajaí, estado de Santa Catarina, Sul do Brasil. Foram discutidos alguns aspectos sobre a história natural e conservação destas espécies.

\section{Materiais e Métodos}

\section{Área de estudo}

A Reserva Biológica Estadual do Sassafrás (RBES) é dividida em: Gleba-1, no município Doutor Pedrinho e a Gleba-2, município Benedito Novo (Figura 1), inseridos na região do Médio Vale do Rio Itajaí. O

FIGURA 1: Mapa do estado de Santa Catarina, destacando as duas glebas da Reserva Biológica Estadual do Sassafrás, sendo A - Gleba Maior e B - Gleba menor.

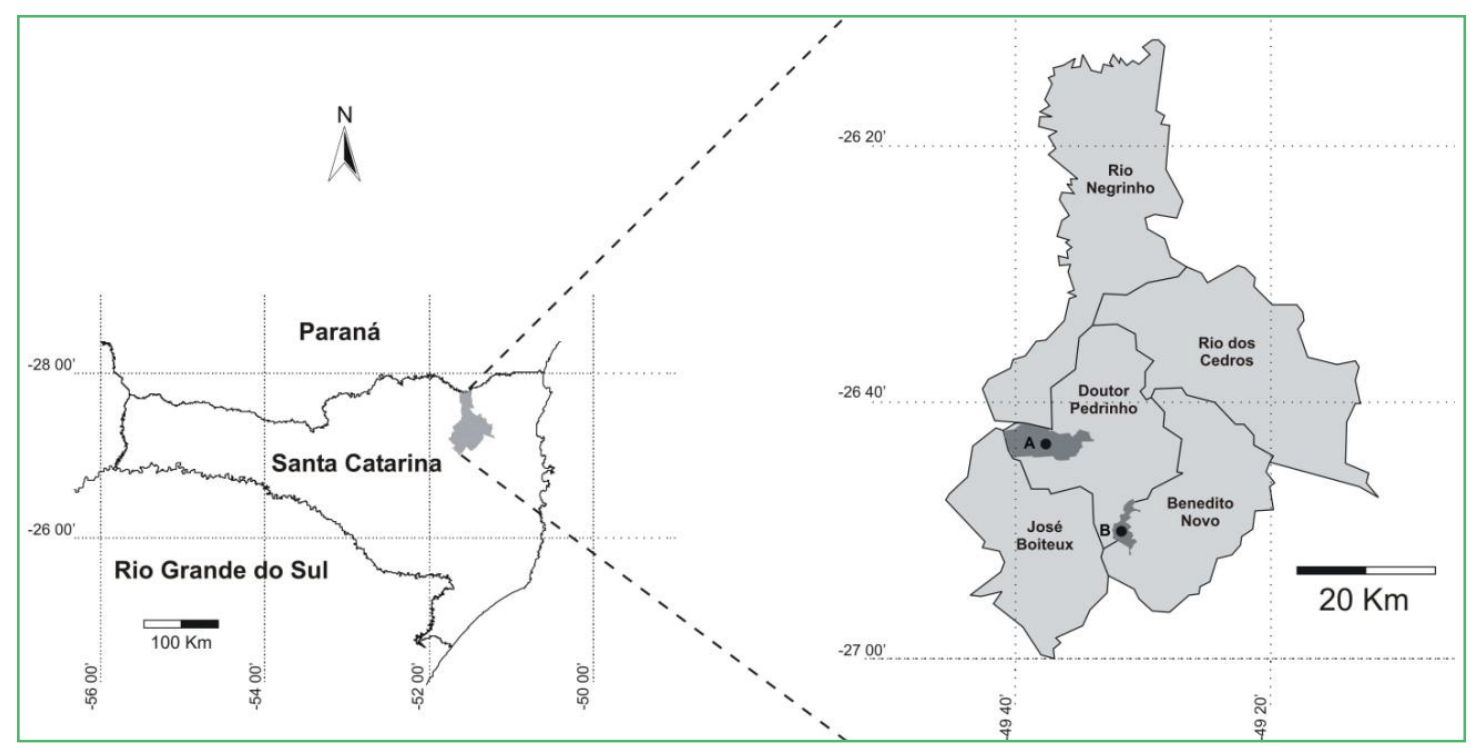


presente trabalho foi realizado na Gleba-1, que possui uma área de 3.682 ha, situada na localidade de Alto Forcação (26² $42^{\prime}$ S, 49 $\left.49^{\circ} \mathrm{W}\right)$, na Serra da Moema. O clima na região é mesotérmico úmido, sem estação seca (Cfa), com pluviosidade de 1.400 a $1.500 \mathrm{~mm}$ anual e temperatura média anual de $19^{\circ} \mathrm{C}$ (GAPLAN, 1986; PELL et al., 2007). O relevo é acidentado, com altitudes variando de 500 a $1000 \mathrm{~m}$.

A RBES se encontra em área de transição entre as Florestas Ombrófila Densa (FOD) e Ombrófila Mista (FOM) (IBGE, 2004), com adensamento de canelasassafrás (Ocotea odorifera), originando o nome da reserva. As áreas amostradas incluem trilhas que atravessam florestas primárias, secundárias, capoeiras e margens de riachos. Também foram registradas espécies em áreas limítrofes da reserva, como sítios e fazendas de reflorestamento com Pinus sp.

\section{Métodos}

A coleta de informações em campo ocorreu entre agosto de 2004 a março de 2008, variando de dois a cinco dias. O inventário foi realizado através de armadilhas fotográficas, visualizações, coleta de animais encontrados mortos, identificação de rastros e captura de pequenos mamíferos através de armadilhas de captura viva, modelo Sherman $(300$ x 80 x $90 \mathrm{~mm})$. O uso de armadilhas fotográficas variou de uma única até seis armadilhas fotográficas Tigrinus ${ }^{\circledR}$ simultaneamente em campo, permanecendo instaladas de 15 a 40 dias.

Os registros através de visualizações, coleta de animais mortos e identificação de rastros ocorreram de forma oportuna durante o percurso das trilhas da RBES, aceiros e estradas da região de entorno. Os animais encontrados mortos, eventualmente vítimas de atropelamento, quando coletados, foram depositados na coleção científica do Laboratório de Biologia Animal, Universidade Regional de Blumenau (FURB), Brasil. Os rastros foram identificados através da utilização de guias de campo (BECKER; DALPONTE, 1999; LIMABORGES; TOMAS, 2004). Não foram descriminadas as espécies de felinos de pequeno porte e cervídeos, através de seus rastros. Estas espécies somente foram confirmadas na área através de registros fotográficos.
Espécies de roedores e marsupiais foram registradas através de captura com 20 armadilhas de captura viva dispostas no solo em área de floresta secundária e coleta de animais encontrados mortos, principalmente durante $o$ fenômeno da ratada que ocorreu no ano de 2007 devido à frutificação de bambus (Chusquea sp.). A identificação de espécies de roedores se realizou através de experiência prévia, guias como Bonvicino et al. (2008) e, quando possível, através de análise citogenética, realizadas de acordo com Ford e Hamerton (1956), com modificações propostas por Sbalqueiro e Nascimento (1996).

Através destas metodologias foi possível verificar alguns aspectos da biologia das espécies de mamíferos registradas. A ordem taxonômica e a nomenclatura para as espécies citadas foram baseadas em Paglia et al. (2012) e Trigo et al. (2013) para o gênero Leopardus.

\section{Resultados}

Em 104 dias em campo, foram registradas 43 espécies de mamíferos terrestres autóctones da RBES, pertencentes a oito ordens: Rodentia (18 espécies), Carnivora (11), Artiodactyla (5), Cingulata (3), Primates (2), Didelphimorphia (2), Pilosa (1) e Perissodactyla (1) (Tabela 1).

O método que obteve o maior número de espécies foi o monitoramento com armadilhas fotográficas (24 espécies) se mostrando uma metodologia eficiente, como já comprovada em estudos anteriores (SRBEKARAÚJO; CHIARELLO, 2005). A utilização das armadilhas fotográficas resultou em um esforço amostral de 2.781 armadilhas/dia onde foram obtidos 381 registros fotográficos de mamíferos de médio e grande porte. As espécies mais abundantes foram: Cerdocyon thous (30,2\%), Eira barbara (13,1\%), Cuniculus paca (11,5\%), Dasypus novemcinctus (10,8\%) e Leopardus pardalis $(10,2 \%)$. Esta metodologia foi responsável pelos registros de Leopardus wiedii e a distinção entre as espécies de cervídeos. Armadilhas de captura viva foram instaladas em seis noites, totalizando um esforço amostral 120 armadilhas/noite. Através deste método foram capturados 27 indivíduos de nove espécies da família Cricetidae. A espécie mais abundante foi Akodon montensis, totalizando 10 indivíduos capturados. todas 
TABELA 1: Mamíferos terrestres registrados na Reserva Biológica Estadual do Sassafrás, durante dos anos de 2004 a 2008, no estado de Santa Catarina, Brasil. Registro: A. Vestígios (rastros e fezes); B. Armadilha fotográfica; C. Visualização; D. Animais encontrados mortos; E. Coleta com armadilhas.

\begin{tabular}{|c|c|c|c|}
\hline Espécie & Registro & $\begin{array}{c}\text { Espécies ameaçadas (FATMA } \\
\text { 2010) }\end{array}$ & $\begin{array}{l}\text { Espécies depositadas em } \\
\text { coleção científica* }\end{array}$ \\
\hline \multicolumn{4}{|l|}{ Didelphimorphia } \\
\hline Didelphis aurita (Wied-Neuwied, 1826) & $\mathrm{B}, \mathrm{D}$ & & $\mathrm{X}$ \\
\hline Philander frenatus (Olfers, 1818) & $\mathrm{B}, \mathrm{E}$ & & $\mathrm{X}$ \\
\hline \multicolumn{4}{|l|}{ Cingulata } \\
\hline Tamandua tetradactyla (Linnaeus, 1758) & $\mathrm{A}, \mathrm{C}$ & & \\
\hline \multicolumn{4}{|l|}{ Pilosa } \\
\hline Cabassous tatouay (Desmarest, 1804) & $\mathrm{A}, \mathrm{B}, \mathrm{D}$ & & $\mathrm{X}$ \\
\hline Dasypus novemcinctus (Linnaeus, 1758) & $\mathrm{A}, \mathrm{B}, \mathrm{D}$ & & $\mathrm{X}$ \\
\hline Dasypus septemcinctus (Linnaeus, 1758) & $\mathrm{B}, \mathrm{D}$ & & $\mathrm{X}$ \\
\hline \multicolumn{4}{|l|}{ Perissodactyla } \\
\hline Tapirus terrestris (Linnaeus, 1758) & A & Em perigo & \\
\hline \multicolumn{4}{|l|}{ Artiodactyla } \\
\hline Mazama americana (Erxleben, 1777) & $\mathrm{B}, \mathrm{C}$ & Em perigo & \\
\hline Mazama gouazoubira (G. Fischer, 1814) & $\mathrm{B}$ & & \\
\hline Mazama nana (Hensel, 1872) & B & Vulnerável & \\
\hline Pecari tajacu (Linnaeus, 1758) & A, B, D & Vulnerável & $\mathrm{X}$ \\
\hline Tayassu pecari (Link, 1795) & $\mathrm{A}, \mathrm{B}, \mathrm{D}$ & Criticamente ameaçada & \\
\hline \multicolumn{4}{|l|}{ Primates } \\
\hline Alouatta guariba (Humboldt, 1812) & $\mathrm{C}$ & Vulnerável & \\
\hline Sapajus nigritus (Goldfuss, 1809) & $\mathrm{C}$ & & \\
\hline \multicolumn{4}{|l|}{ Carnívora } \\
\hline Cerdocyon thous (Linnaeus, 1766) & $\mathrm{A}, \mathrm{B}, \mathrm{C}, \mathrm{D}$ & & $\mathrm{X}$ \\
\hline Leopardus pardalis (Linnaeus, 1758) & $\mathrm{A}, \mathrm{B}, \mathrm{C}$ & Em perigo & \\
\hline Leopardus guttulus (Schreber, 1775) & $\mathrm{B}, \mathrm{C}$ & & $\mathrm{X}$ \\
\hline Leopardus wiedii (Schinz, 1821) & $\mathrm{B}$ & & \\
\hline Puma concolor (Linnaeus, 1771) & A, B & Vulnerável & \\
\hline Puma yagouaroundi (É. Geoffroy, 1803) & $\mathrm{B}, \mathrm{C}$ & & \\
\hline Eira barbara (Linnaeus, 1758) & $\mathrm{A}, \mathrm{B}, \mathrm{C}, \mathrm{D}$ & & $\mathrm{X}$ \\
\hline Galictis cuja (Molina, 1782) & $\mathrm{A}, \mathrm{B}, \mathrm{C}$ & & $\mathrm{X}$ \\
\hline Lontra longicaudis (Olfers, 1818) & A & & \\
\hline Nasua nasua (Linnaeus, 1766) & A, B, D & & $\mathrm{X}$ \\
\hline Procyon cancrivorus (G. Cuvier, 1798) & $\mathrm{A}, \mathrm{B}, \mathrm{C}$ & & \\
\hline \multicolumn{4}{|l|}{ Rodentia } \\
\hline Cavia aperea (Erxleben, 1777) & C, D & & $\mathrm{X}$ \\
\hline Hydrochoerus hydrochaeris (Linnaeus, 1766) & $\mathrm{A}, \mathrm{B}, \mathrm{C}$ & & \\
\hline Akodon montensis (Thomas, 1913) & E & & $\mathrm{X}$ \\
\hline $\begin{array}{l}\text { Akodon paranaensis (Christoff, Fagundes, Sbalqueiro, } \\
\text { Mattevi \& Yonenaga-Yassuda, 2000) }\end{array}$ & $\mathrm{E}$ & & $\mathrm{X}$ \\
\hline Akodon serrensis (Thomas, 1902) & $\mathrm{E}$ & & $\mathrm{X}$ \\
\hline Delomys dorsalis (Hensel, 1872) & E, D & & $\mathrm{X}$ \\
\hline Euryoryzomys russatus (Wagner, 1848) & E, D & & $\mathrm{X}$ \\
\hline Oligoryzomys nigripes (Olfers, 1818) & E, D & & $\mathrm{X}$ \\
\hline Oxymycterus sp. & E, D & & $\mathrm{X}$ \\
\hline Sooretamys angouya (G. Fischer, 1814) & E, D & & $\mathrm{X}$ \\
\hline Thaptomys nigrita (Lichtenstein, 1829) & C, E, D & & $\mathrm{X}$ \\
\hline Cuniculus paca (Linnaeus, 1766) & $\mathrm{A}, \mathrm{B}, \mathrm{C}$ & Vulnerável & \\
\hline Dasyprocta azarae (Lichtenstein, 1823) & $\mathrm{A}, \mathrm{C}$ & & \\
\hline Euryzygomatomys spinosus (G. Fischer, 1814) & $\mathrm{D}$ & & $\mathrm{X}$ \\
\hline Kannabateomys amblyonyx (Wagner, 1845) & $\mathrm{C}$ & & \\
\hline Myocastor coypus (Molina, 1782) & $\mathrm{C}$ & & \\
\hline Coendou villosus (F. Cuvier, 1823) & $\mathrm{B}, \mathrm{D}$ & & \\
\hline Guerlinguetus ingrami (Thomas, 1901) & $\mathrm{B}, \mathrm{C}$ & & \\
\hline
\end{tabular}

* Informações sobre espécies coletadas no anexo 1. 
identificadas através de análise citogenética: Akodon montensis $(2 \mathrm{n}=24$ e 25$)$, Akodon paranaensis $(2 \mathrm{n}=44)$, Akodon serrensis $(2 \mathrm{n}=46)$, Delomys dorsalis $(2 \mathrm{n}=82)$, Oligoryzomys nigripes $(2 \mathrm{n}=62)$, Sooretamys angouya $(2 \mathrm{n}=58)$, Euryoryzomys russatus $(2 \mathrm{n}=80)$, Oxymycterus sp. $(2 \mathrm{n}=52)$ e Thaptomys nigrita $(2 \mathrm{n}=52)$.

Além das espécies nativas, três espécies exóticas foram registradas no presente estudo, Mus musculus e Rattus rattus na ordem Rodentia e Lepus capensis representante da ordem Lagomorpha.

\section{Discussão}

Através do inventário realizado, a UC Reserva Biológica Estadual do Sassafrás obteve uma elevada riqueza de espécies de mamíferos. Considerando que o estado de Santa Catarina possui 81 espécies de mamíferos terrestres não voadores (CHEREM et al., 2004), na RBES encontram-se 53\% destas espécies. Comparando os dados deste trabalho com outros estudos realizados no sul do Brasil envolvendo mamíferos terrestres (Tabela 2), mesmo considerando que estes estudos foram realizados em diferentes áreas e com metodologias distintas, a RBES apresentou expressiva riqueza de espécies, apresentando o maior número de espécies da Ordem Artiodactyla.
Neste trabalho, as Ordens Didelphimorphia e Rodentia foram consideradas sub-amostradas, pois seria necessário um maior número de armadilhas/noite, direcionado para a captura destas espécies, utilizando-se de armadilhas dispostas no subosque e armadilhas de intercepção e queda. Apesar do baixo esforço, o registro de Akodon serrensis é um dos poucos conhecidos para o estado de Santa Catarina, conforme Testoni et al. (2012).

Em relação às ameaças, cinco espécies registradas neste estudo são consideradas ameaçadas de extinção a nível nacional (MMA, 2003) e nove considerados ameaçadas de extinção a nível estadual, sendo Tayassu pecari considerada criticamente ameaçada, Leopardus pardalis, Tapirus terrestris e Mazama americana consideradas em perigo, Alouatta guariba, Puma concolor, Pecari tajacu, Mazama nana e Cuniculus paca consideradas vulneráveis (FATMA, 2010). Os autores consideram a nível regional, que o desmatamento e caça furtiva representam as principais ameaças para mastofauna, pois espécies como Myrmecophaga tridactyla, Ozotoceros bezoarticus (TORTATO; ALTHOFF, 2011) e Speothos venaticus (CIMARDI, 1996) outrora ocorrentes na região amostrada não foram registradas neste estudo, representando indícios de que esta região já sofreu extinções locais.

Com os resultados aqui apresentados, a RBES se mostra de grande importância para a conservação

TABELA 2: Número de espécies de mamíferos (exceto mamíferos marinhos e morcegos) registrados em estudos recentes realizados no Sul do Brasil.

\begin{tabular}{|c|c|c|c|c|c|c|c|c|}
\hline \multirow{2}{*}{ ORDEM } & \multicolumn{8}{|c|}{ Referência } \\
\hline & $\mathbf{A}$ & B & $\mathrm{C}$ & D & $\mathbf{E}$ & $\mathbf{F}$ & $\mathbf{G}$ & H \\
\hline Didelphimorphia & 3 & 4 & 4 & 2 & 4 & 1 & 10 & 2 \\
\hline Pilosa & 1 & 1 & 1 & - & 1 & 1 & 4 & 3 \\
\hline Cingulata & 2 & 1 & 3 & - & 4 & 2 & 1 & 1 \\
\hline Primates & 1 & 1 & 1 & 1 & 2 & 2 & 2 & 2 \\
\hline Carnívora & 8 & 11 & 4 & 7 & 10 & 11 & 12 & 11 \\
\hline Perissodactyla & - & 1 & - & - & - & - & 1 & 1 \\
\hline Artiodactyla & 2 & 2 & - & 1 & 2 & 3 & 3 & 5 \\
\hline Lagomorpha & 1 & - & - & - & 1 & 1 & - & - \\
\hline Rodentia & 14 & 10 & 12 & 8 & 19 & 5 & 26 & 18 \\
\hline TOTAL & 32 & 31 & 25 & 19 & 43 & 26 & 59 & 43 \\
\hline
\end{tabular}

Referências: A - Wallauer et al. (1999); B - Quadros e Cáceres (2001); C - Graipel et al. (2001); D - Cáceres (2004); E - Kasper et al. (2007); F - Miranda et al. (2008); G - Cherem et al. (2011); H - Este estudo. 
da mastofauna de Santa Catarina. A ocorrência de nove espécies ameaçadas a nível estadual, destacando o registro de Tayassu pecari, é um indicativo das boas condições que se encontra esta UC. Uma gestão adequada pelo órgão competente e a manutenção da conectividade desta UC com áreas preservadas em seu entorno são necessárias para que a RBES continue considerada mantenedora da biodiversidade de espécies de mamíferos de Santa Catarina.

\section{Agradecimentos}

Os autores agradecem Adrian Eisen Rupp, Carlos Zimmermann, Gregory Thom e Silva, Jairo C. dos Santos, José Correa, Marcos A. Tortato por toda ajuda em campo. A Tigrinus Equipamentos para Pesquisa, Fundação do Meio Ambiente do estado de Santa Catarina (FATMA), Modo Battistella Reflorestamento - MOBASA e Aderbal Domingos Tortato pelo auxilio financeiro e logístico.

\section{Referências}

BECKER, M.; DALPONTE, J. M. Rastros de mamíferos silvestres brasileiros. Brasília: Editora UNB, 1999. 180 p.

BONVICINO, C. R.; DE OLIVEIRA, J. A.; D'ANDREA, P. S. Guia de roedores do Brasil, com chaves para gêneros baseadas em caracteres externos. Rio de Janeiro: Centro Pan-Americano de Febre Aftosa - OPAS/OMS, 2008. 120 p.

BRITO, D.; OLIVEIRA, L. C.; OPREA, M.; MELO, M. An overview of Brazilian mammals: trends, biases and future directions. Zoologia, Curitiba, v. 26, n. 1, p. 67-73, 2009.

CÁCERES, N. C. Occurrence of Conepatus chinga (Molina) (Mammalia, Carnivora, Mustelidae) and other terrestrial mammals in the Serra do Mar, Paraná, Brazil. Revista Brasileira de Zoologia, Curitiba, v. 21, n. 3, p. 577-579, 2004.

CÁCERES, N. C.; CHEREM, J. J.; GRAIPEL, M. E. Distribuição geográfica de mamíferos terrestres da região Sul do Brasil. Ciência \& Ambiente, Santa Maria, v. 35, p. 167-180, 2007

CANALE, G. R.; PERES, C. A.; GUIDORIZZI, C. E.; GATTO, C. A. F.; KIERULFF, M. C. M. Pervasive defaunation of forest remnants in a tropical biodiversity hotspot. PLOS ONE, Cambridge, v. 7, n. 8, e41671, 2012

CHEREM, J. J.; GRAIPEL, M. E.; TORTATO, M.; ALTHOFF, S.; BRÜGGEMANN, F.; MATOS, J.; VOLTOLINI, J. C.; FREITAS, R.; ILLENSEER, R.; HOFFMANN, F.; GHIZONIJR, I. R.; BEVILACQUA, A.; REINECKE, R.; SALVADOR, C. H.; FILIPPINI, A.; FURNARI, N.; ABATI, K.; MORAES, M.; MOREIRA, T.; OLIVEIRA-SANTOS, L. G. R.; KUHNEN, V.; MACCARINI, T.; GOULART, F.; MOZERLE, H.; FANTACINI,
F.; DIAS, D.; PENEDO-FERREIRA, R.; VIEIRA, B. P.; SIMÕESLOPES, P. C. Mastofauna terrestre do Parque Estadual da Serra do Tabuleiro, estado de Santa Catarina, sul do Brasil. Biotemas, Florianópolis, v. 24, n. 3, p. 73-84, 2011.

CHEREM, J. J.; SIMÕES-LOPES, P. C.; ALTHOFF, S. L.; GRAIPEL, M. E. Lista dos mamíferos do estado de Santa Catarina, sul do Brasil. Mastozoologia Neotropical, Mendoza, v. 11, n. 2, p. 151-184, 2004.

CHIARELLO, A. G. Effects of fragmentation of the Atlantic forest on mammal communities in south-eastern Brazil. Biological Conservation, Boston, v. 89, p. 71-82, 1999.

CIMARDI, A. V. Mamíferos de Santa Catarina. Florianópolis: FATMA. 1996. $302 \mathrm{p}$

COSTA, L. P.; LEITE, Y. L. R.; MENDES, S. L.; DITCHFIELD, A. D. Mammal conservation in Brazil. Conservation Biology, San Francisco, v. 19, n. 3, p. 672-679, 2005.

FATMA-FUNDAÇÃO DE MEIOAMBIENTE. Lista das espécies da fauna ameaçada de extinção em Santa Catarina. 2010. 57 p. Disponível em: <http://www.fatma.sc.gov.br/upload/Fauna/ relat9500rio_t9500cnico_final_lista_esp9500cies_amea9500adas. pdf $>$. Accesso em: 24 nov. 2013.

FORD, C. E.; HAMERTON J. L. A colchicine hypotonic citrate squash sequence for mammalian chromosomes. Stain Technology, Cambridge, v. 31, p. 247-251, 1956.

GAPLAN. Atlas de Santa Catarina. Rio de Janeiro: Aerofoto Cruzeiro, 1986. 173 p.

IBGE - INSTITUTO BRASILEIRO DE GEOGRAFIA E ESTATÍSTICA. Mapa de biomas e de vegetação. Brasília: IBGE, 2004. Disponível em: <http://www.ibge.gov.br/home/presidencia/ noticias/21052004biomas.shtm>.

KASPER, C. B.; MAZIM, F. D.; SOARES, J. B. G.; OLIVEIRA, T. G.; FABIÁN, M. E. Composição e abundância relativa dos mamíferos de médio e grande porte no Parque Estadual do Turvo, Rio Grande do Sul, Brasil. Revista Brasileira de Zoologia, Curitiba, v. 24, n. 4, p. 1087-1100, 2007.

LIMA-BORGES, P. A.; TOMAS, W. M. Guia de rastros e outros vestígios de mamíferos do Pantanal. Corumbá: Embrapa Pantanal, 2004. 148 p.

MAZZOLLI, M. Loss of historical range of jaguars in southern Brazil. Biodiversity and Conservation, New York, v. 18, n. 6, p. 1715-1717, 2008.

MEDEIROS, J. D. Mata Atlântica em Santa Catarina. In: SCHÄFFER, W. B.; PROCHNOW, M. (Ed.). A Mata Atlântica e você: como preservar, recuperar e se beneficiar da mais ameaçada floresta brasileira. Brasília: Apremavi, 2002. p. 103-109.

MIRANDA, J. M. D.; MORO-RIOS, R. F.; PASSOS, F. C. Contribuição ao conhecimento dos mamíferos dos Campos de Palmas, Paraná, Brasil. Biotemas, Florianópolis, v. 21, n. 2, p. $97-$ 103, 2008.

MMA - MINISTÉRIO DO MEIO AMBIENTE. Lista oficial das espécies da fauna brasileira ameaçadas de extinção. 2003. Instrução Normativa no 3, de 27 de maio de 2003. Diário Oficial da República Federativa do Brasil, Brasilia, DF. Seção 1, 101:88-97. Disponível em: <http://www..gov.br/estruturas/179/ arquivos/179_05122008034002.pdf>. Acesso em: 24 nov.2013. 
PAGLIA, A. P.; FONSECA, G. A. B.; RYLANDS, A. B.; HERRMANN, G.; AGUIAR, L. M. S.; CHIARELLO, A. G.; LEITE, Y. L. R.; COSTA, L. P.; SICILIANO, S.; KIERUFF, M. C. M.; MENDES, S. L.; TAVARES, V. C.; MITTERMEIER, R. A.; PATTON, J. J. Annotated checklist of Brazilian mammals. 2. ed. Occasional Papers in Conservation Biology, $n^{-}$6. Arlington: Conservation International, 2012. 76 p.

PELL, M. C.; FINLAYSON B. L.; MCMAHON T. A. Updated world map of the Köppen-Geiger climate classification. Hydrology and Earth System Sciences, Delft, v. 11, p. 1633-1644, 2007.

QUADROS, J.; CÁCERES, N. C. Ecologia e conservação de mamíferos na Reserva Volta Velha, SC, Brasil. Acta Biologica Leopoldensia, São Leopoldo, v. 23, p. 213-224, 2001.

SBALQUEIRO, I. J.; NASCIMENTO A. P. Occurrence of Akodon cursor (Rodentia, Cricetidae) with 14, 15 and 16 chromosome cytotypes in the same geographic area in southern Brazil. Brazilian Journal of Genetics, Ribeirão Preto, v. 19, p. 565-569, 1996.

SRBEK-ARAUJO, A. C.; CHIARELLO, A. G. Is camera-trapping na efficient method for surveying mammals in Neotropical forests? A case study in south-eastern Brazil. Journal of Tropical Ecology, Cambridge, v. 21, p. 121-125, 2005.

TESTONI, A. F.; FUMIS, J.; ALTHOFF, S. L.; TORTATO, F. R.; CHEREM, J. J. Akodon serrensis Thomas, 1902 (Mammalia: Rodentia: Sigmodontinae): records in Santa Catarina state, southern Brazil. Check List, Rio Claro, v. 8, n. 6, p. 1344-1346, 2012.

TORTATO, F. R.; ALTHOFF, S. L. Mammalia, Myrmecophagidae, Myrmecophaga tridactyla (Linnaeus, 1758) and Cervidae, Ozotoceros bezoarticus (Linnaeus, 1758): contribution to the knowledge of the historical distribution in Santa Catarina, southern Brazil. Check List, Rio Claro, v. 7, n. 2, p. 146-148, 2011.

TRIGO, T. C.; SCHNEIDER, A.; OLIVEIRA, T. G.; LEHUGEUR, L. M.; SILVEIRA, L.; FREITAS, T. R. O.; EIZIRIK, E. Molecular Data Reveal Complex Hybridization and Cryptic Species of Neotropical Wild Cat. Current Biology, London, v. 23, n. 24, p. 2528-2533, 2013.

WALLAUER, J. P.; BECKER, M.; MARINS-SÁ, L. G.; LIERMANN, L. M.; PERRETO, S. H.; SCHERMACK, V. Levantamento dos mamíferos da Floresta Nacional de Três Barras - Santa Catarina. Biotemas, Florianópolis, v. 13, n. 1, p. 103-127, 2000 .

\section{ANEXO 1}

Exemplares coletados na Reserva Biológica Estadual do Sassafrás e entorno e depositados na coleção científica da Fundação Universidade Regional de Blumenau (FURB).

Didelphis aurita (FURB 12142)

Philander frenatus (FURB 12166)

Cabassous tatouay (FURB 12633)

Dasypus novemcinctus (FURB 12635)

Dasypus septemcinctus (FURB 12510)

Pecari tajacu (FURB 12245)

Cerdocyon thous (FURB 12069)

Leopardus guttulus (FURB 12787)

Eira Barbara (FURB 12434)

Galictis cuja (FURB 12278)

Nasua nasua (FURB 12246)

Cavia aperea (FURB 12270)

Akodon montensis (FURB 12134, FURB 12135, FURB 12138, FURB 12140, FURB 12141, FURB 12163, FURB 12164, FURB 12248, FURB 12249, FURB 12251)

Akodon paranaensis (FURB 12252)

Akodon serrensis (FURB 12135, FURB 12139, FURB 12248, FURB 12249, FURB 12702)

Delomys dorsalis (FURB 12098)

Euryoryzomys russatus (FURB 12137)

Oligoryzomys nigripes (FURB 12102, FURB 12148)

Oxymycterus sp. (FURB 12250, FURB 12558, FURB 12721)

Sooretamys angouya (FURB 12165, FURB 12268)

Thaptomys nigrita (FURB 12099)

Euryzygomatomys spinosus (FURB 12092) 